\title{
A STUDY DESCRIBING THE USAGE PATTERN OF ANTIMICROBIALS WITH INSIGHTS INTO ANTIMICROBIAL DRUG-DRUG INTERACTIONS AIMONG ADULT POPULATION IN HOSPITAL SETTINGS
}

KEY WORDS: Antimicrobials, drug-drug interaction, monotherapy
Tabassum

Fathima

Prukruthi R

Manikandan $\bar{A}$

Muhammed

Ramees P I

\section{Leena Pavitha P*}

C.LBaid Metha College Of Pharmacy, Chennai (Pharm.D Intern)

C.L Baid Metha College Of Pharmacy, Chennai (Pharm.D Intern)

C.L Baid Metha College Of Pharmacy, Chennai (Pharm.D Intern)

C.L Baid Metha College Of Pharmacy, Chennai (Pharm.D Intern)

Sri Ramachandra Institute Of Higher Education And Research, Chennai (Ph. D scholar).*Corresponding Author

The study aims to describe the use of antimicrobials among adult population in hospital settings with emphasis on the antimicrobial therapy provided and potential antimicrobial drug-drug interactions identified. 108 adult patients who were prescribed antimicrobials were considered for this retrospective study which was carried out over a period of 3 months. It was identified that antimicrobials prescribed were largely antibacterial (91.2\%) with Piperacillin +

터 Tazobactam (24 times) and Cefuroxime (15 times) being the most commonly prescribed antimicrobials on treatment

0 and discharge, respectively. Upon assessing the antimicrobial therapy, it was identified that antimicrobials were predominantly prescribed empirically $(57.4 \%)$ and monotherapy was observed more, both on treatment (52.8\%) and discharge $(47.2 \%)$. A total of 79 different potential antimicrobial drug-drug interactions were identified, out of which, 64.6\% were major interactions. Ciprofloxacin + Metronidazole drug-drug interaction was the most common drug interaction observed 6 times, whereas clarithromycin and ciprofloxacin caused the greatest number of interactions with a frequency of 10 instances each. Ondansetron was the non-antimicrobial drug that caused the greatest number of drug interactions $(21.2 \%)$. The present study reinforces that antibiotics and other antimicrobials are a group of very commonly prescribed medications in the hospital with a variety of indications. An important, but often unheeded aspect of therapy is antimicrobial interactions with other drugs, which this study has highlighted.

\section{INTRODUCTION:}

Antimicrobials have long been considered as the 'magic bullet' that would end an infectious disease. Until the 20th century, infections that are now considered straight forward to treat, such as Pneumonia and Diarrhoea, were the key factors of mortality. Antimicrobials, since their discovery, are now considered to be one of the most successful forms of chemotherapeutic agents that can cure and not just palliate a disease.

Since infections remain a major problem in medical practice, rational treatment with appropriate antimicrobials is of prime importance. ${ }^{[1]}$ Resistance of the infectious microorganism towards antimicrobials is a serious concern. According to the WHO, antimicrobial resistance is one of the world's top 10 global health threats. ${ }^{[2]}$ The impact of resistance in India is likely to be on the higher side as it has the highest number of MDR-TB cases reported. Gram-positive and Gram-negative organisms have shown high resistance towards Fluoroquinolones, Carbapenems, and Colistin. ${ }^{[3]}$ Therefore, it is essential to select antimicrobials based on culture reports of the specimen sent for microbiological testing.

From the terminological point of view, an appropriate treatment is the one that is active against pathogens. Proper treatment refers not only to its appropriateness but also to its rational prescribing, following the current treatment guidelines. ${ }^{[4]}$

According to the WHO, presumptive (empirical) therapy is a onetime treatment (generally with a broad-spectrum antimicrobial) given for a presumed infection in patients while waiting for the culture sensitivity report which could take more than 72 hours to be generated. ${ }^{[4]}$ Empirical therapy can be initiated in critically ill patients where antimicrobials are selected according to the clinical symptoms exhibited by the patient. Prophylaxis of antimicrobials should be patientspecific and indication-specific to provide a cost-effective treatment, prevent the emergence of resistance and adverse drug events. ${ }^{[5]}$

Monotherapy is preferred wherever possible to avoid antagonism between two drugs and to prevent the occurrence of any adverse events in patients. Combined use of two or more antimicrobials is not ordinarily recommended. However, they may be combined under certain conditions: to achieve synergy against a single organism, to delay the development of or to overcome drug resistance, when an etiological diagnosis is unknown, when the infection is severe, when the body defence is poor, and for reducing the chances of superinfection.

The concomitant use of two or more antimicrobials may have contraindications due to drug-drug interactions and should not be used when these are present. Potential drug-drug interactions refer to the possibility of a drug altering the effects of another drug when used simultaneously. ${ }^{[6]}$ Since antimicrobials constitute a large group of drugs that are very commonly used, there are high chances of observing significant potential drug-drug interactions in the hospital and ambulatory care settings. In the outpatient settings, oral antimicrobials are the major cause of drug-drug interactions as parenteral antimicrobials are rarely prescribed. The increasing medication burden in patients-the number of medications or pills taken daily by the patient for treatment of their disease- and disregarding the patient's crucial perspectives has surged the likelihood of these drug interactions.

\section{MATERIALS AND METHODS:}

The study design is retrospective, carried out over a period of 3 months in a tertiary care hospital. 108 adult hospitalised patients were included in the study. The patients had received at least one antimicrobial upon admission, discharge or both. Outpatients, pregnant and lactating women, and transplant patients had been excluded from the study. Data were 
maintained in a suitable proforma. Patient-specific details such as age, gender, duration of stay, chief complaints, past medical and medication histories and present illness were collected. Antimicrobial-specific details such treatment given during hospital stay and discharge, dose and duration of antimicrobial use were compiled. Data analysis was performed using MS Excel. The potential drug-drug interactions between antimicrobials and with other drugs were assessed and classified according to the MICROMEDEX database.

\section{RESULTS:}

Out of the 108 adult patients, 67 (62\%) patients were male and $41(38 \%)$ were female. Patients aged between 50 and 59 years $(20.4 \%)$ were the largest age group receiving antimicrobials. Diabetes was found to be the most common medical comorbid condition followed by systemic hypertension with an occurrence in $37(18.5 \%)$ and 22 (16.5\%) patients, respectively.

62 patients $(57.4 \%)$ were found to have more than one presenting illness on admission with 9 (5.0\%) of them diagnosed with urinary tract infections.

43 different antimicrobials had been used during treatment whereas 36 antimicrobials had been prescribed during discharge. Categories of antimicrobials identified in the study are given in Table 1 .

\section{Table: 1 Categories of antimicrobials}

\begin{tabular}{|c|c|c|}
\hline $\begin{array}{c}\text { Categories of } \\
\text { Antimicrobials }\end{array}$ & $\begin{array}{c}\text { Frequency } \\
\text { (n=308) }\end{array}$ & $\begin{array}{c}\text { Percentage } \\
\text { (\%) }\end{array}$ \\
\hline Anti-Bacterial & $\mathbf{2 8 1}$ & $\mathbf{9 1 . 2}$ \\
\hline Anti-Fungal & 11 & 3.6 \\
\hline Anti-Malarial & 4 & 1.3 \\
\hline Anti-Viral & 12 & 3.9 \\
\hline
\end{tabular}

Piperacillin + Tazobactam was the most commonly prescribed antimicrobial with a frequency of 24 times followed by Cefoperazone + Sulbactam with 22 times. Cefuroxime and Ceftriaxone came third and fourth with a frequency of 20 and 16 times, respectively.

Upon discharge, 37 patients did not receive any antimicrobial. Upon discharge, Cefuroxime was the most frequent antimicrobial prescribed (15 times) followed by Doxycycline and Ciprofloxacin (6 times each).

Antimicrobials were prescribed empirically to $62(57.4 \%)$ patients while prophylaxis was observed in 34 (31.5\%) patients. $12(11.1 \%)$ patients had received both empirical and prophylactic therapy.

The patterns of monotherapy and combination therapy are given in Table 2.

Table 2:Patterns of Monotherapy and Combination therapy

\begin{tabular}{|c|c|c|}
\hline Parameter & Frequency (n=108) & Percentage (\%) \\
\hline \multicolumn{3}{|c|}{ On Treatment } \\
\hline Monotherapy & $\mathbf{5 7}$ & $\mathbf{5 2 . 8}$ \\
\hline Combination & 51 & 47.2 \\
\hline \multicolumn{3}{|c|}{ On Discharge } \\
\hline Monotherapy & $\mathbf{5 1}$ & $\mathbf{4 7 . 2}$ \\
\hline Combination & 20 & 18.5 \\
\hline None & 37 & 34.2 \\
\hline
\end{tabular}

79 different types of potential antimicrobial drug-drug interactions were identified with a total frequency of 121 times. 51 (64.6\%) were major followed by 21 (26.6\%) moderate interactions. Contraindicated drug interactions were infrequent with 4 interactions $(5.1 \%)$ identified. Antimicrobials causing drug interactions are given in Figure 1 and the most common drug interactions are given in table 3 .

\section{ANTIMICROBLALS INVOLVED IN DRUG INTERACTIONS}

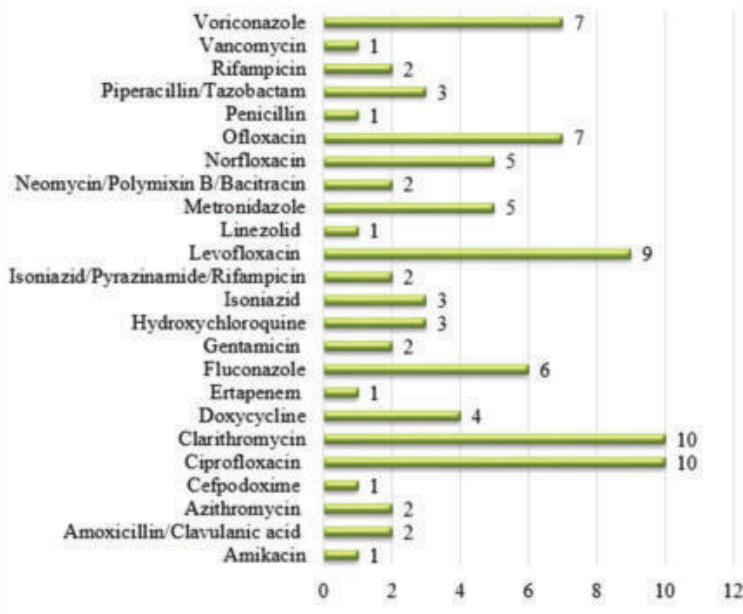

Figure: 1 Antimicrobials causing drug interactions

Table 3: Drug-Drug interaction

\begin{tabular}{|c|c|c|}
\hline Drug Interaction & Severity & $\begin{array}{c}\text { Frequency } \\
(\mathbf{n = 1 2 1 )}\end{array}$ \\
\hline Ciprofloxacin + Metronidazole & Major & $\mathbf{6}$ \\
\hline Clarithromycin + Ondansetron & Major & 5 \\
\hline $\begin{array}{c}\text { Clarithromycin + Budesonide/ } \\
\text { Formoterol }\end{array}$ & Major & 4 \\
\hline Metronidazole + Ondansetron & Major & 4 \\
\hline Azithromycin + Ondansetron & Major & 3 \\
\hline Ciprofloxacin + Ondansetron & Major & 3 \\
\hline Fluconazole + Pantoprazole & Moderate & 3 \\
\hline Levofloxacin + Metformin & Major & 3 \\
\hline
\end{tabular}

Drug interactions between two different antimicrobials had a frequency of 17 times. It was observed that among the nonantimicrobial classes of drugs, ondansetron had the highest number of interactions with a frequency of $22(21.2 \%)$ times.

\section{DISCUSSION:}

This study was conducted with the main intention of observing the usage pattern of antimicrobials, to provide insights on antimicrobial therapy and identify interactions between antimicrobials and other drugs. The study is neither confined to a specific population nor to a specific therapy, rather includes generalized ones, so that there would be a cognizance of antimicrobials used in all conditions.

The frequent use of antimicrobials among the older population may be attributed to lifestyle modifications, degenerative changes and decline in the immune response, particularly cell mediated immunity. ${ }^{\left[{ }^{7]}\right.}$ Our study found that infections were the most common presenting illness with urinary tract infections being the most common type of infection. This observation adheres to the belief of using antimicrobials as and when required in infectious patients. The higher percentage of empirical use corresponds with the practice of prescribing based on the presence of signs and symptoms, suspecting, or confirming an infection, rather than giving for prevention.

The most common combination of antimicrobials observed was the prescribing of a beta lactam with another beta lactam (13 times) followed closely by the combination of a beta lactam with a macrolide (12 times). Other combinations observed include a beta lactam with rifamycin (9 times), fluoroquinolone with imidazole and tetracycline with a beta lactam (5 times each). This closely corresponds to the study by Lodise PT et al., where the authors compared the efficacy of the combination of beta lactams with macrolides against fluoroquinolone monotherapy and found that the former was more efficacious than the latter. ${ }^{[8]}$ 
Cephalosporins had the highest frequency among monotherapy followed by the fixed dose combination of beta lactam and beta lactamase inhibitor on treatment as well as discharge. The study result is supported by two studies conducted by Wong $\mathrm{D}$ et al., and Sutter TS et al., while the latter conducted the study exclusively on plasmodium Aeruginosa. ${ }^{[9,10]}$

Theoretically, the possibility for drug interactions to occur is over $50 \%$ when a patient is receiving five medications, and the probability increases to $100 \%$ when seven drugs are used. ${ }^{[1]}$

It has been reported that the prevalence of potential drugdrug interactions in hospitalized patients is approximately $60 \%$ indicating that drug-drug interactions are a relatively common phenomenon. ${ }^{[12]}$ However, only a small proportion $(<5 \%)$ of them cause clinically important adverse drug events (ADEs) ${ }^{[13]}$ Our study identified a large number of potential drug-drug interactions whose clinical significance, unfortunately, is unknown. However, it does shed light on the prevalence of drug-drug interactions, specifically with antimicrobials. Major antimicrobial drug-drug interactions had the highest frequency with fluoroquinolones being the class of antimicrobials causing the most interactions. A multicentre point-prevalence study by Kuscu F et al., revealed similar results where $38 \%$ of major drug-drug interactions were due to antimicrobials. ${ }^{[14]}$

\section{CONCLUSION:}

Antimicrobials are a group of very commonly prescribed medications in hospital settings. Initially prescribed only for suspected or confirmed infectious diseases, these drugs are now prescribed for other uses such as surgical prophylaxis, hepatic encephalopathy prevention etc. Our study has reflected the prescribing of different antimicrobials in a hospital setting in India. It has provided insights on the monotherapy and various combinations of antimicrobials that are routinely used against infections. Inputs on empirical and prophylactic prescribing were described. We have also provided insights into antimicrobial drug-drug interactions to shed light on this increasingly important topic in optimising patient care.

\section{REFERENCES:}

1. Tripathi KD. Essentials of Medical Pharmacology. 7th ed. New Delhi: Jaypee Brothers Medical Publishers (P) Ltd; 2015. Chapter 49: Antimicrobial Drugs: General Considerations; p. 688-703.

2. World Health Organization. (2020, December 24). 10 global health issues to track in 2021. https://www.who.int/news-room/spotlight/10-global-healthissues-to-track-in-2021.

3. Chandra, S., Prithvi, P.P.R., Srija, K., Jauhari, S., \& Grover, A. (2020). Antimicrobial resistance: Call for rational antibiotics practice in India. Journal of family medicine and primary care, 9(5),2192-9.

4. Leekha, S., Terrell, C.L., \& Edson, R.S. (2011). General principles of antimicrobial therapy. Mayo Clinic proceedings, 86(2), 156-67.

5. Enzler, M.J., Berbari, E., \& Osmon, D.R. (2011). Antimicrobial prophylaxis in adults. Mayo Clinic proceedings, 86(7), 686-701.

6. Alvim, M.M., Silva, L.A., Leite, I.C., \& Silvério, M.S. (2015). Adverse events caused by potential drug-drug interactions in an intensive care unit of a teaching hospital. Revista Brasileira de terapia intensiva, 27(4), 353-9.

7. Gardner,I.D. (1980).The effect of aging on susceptibility to infection. Reviews of infectious diseases, 2(5), 801-10.

8. Lodise, T.P., Kwa, A., Cosler, L., Gupta, R., \& Smith, R.P. (2007). Comparison of beta-lactam and macrolide combination therapy versus fluoroquinolone monotherapy in hospitalized Veterans Affairs patients with communityacquired pneumonia. Antimicrobial agents and chemotherapy, 51(11), 397782.

9. Wong,D.,Wong,T., Romney, M., \& Leung,V. (2016). Comparison of outcomes in patients with methicillin-susceptible Staphylococcus aureus (MSSA) bacteremia who are treated with $\square$-lactam vs vancomycin empiric therapy: a retrospective cohort study. BMC infectious diseases, 16, 224

10. Tschudin-Sutter, S., Fosse, N., Frei, R., \& Widmer, A.F. (2018). Combination therapy for treatment of Pseudomonas aeruginosa bloodstream infections. PloS one, 13(9), e0203295.

11. Brahmankar DM, Jaiswal SB. Biopharmaceutics and Pharmacokinetics - A Treatise, 3rd ed.Delhi:Vallabh Prakashan;2015.

12. Egger, S.S., Drewe, J., \& Schlienger, R.G. (2003). Potential drug-drug interactions in the medication of medical patients at hospital discharge. European journal of clinical pharmacology, 58(11),773-8.

13. Krähenbühl-Melcher, A., Schlienger, R., Lampert, M., Haschke, M., Drewe,J., \& Krähenbühl, S. (2007). Drug-related problems in hospitals: a review of the recent literature. Drug safety, 30(5),379-407.

14. Kuscu, F., Ulu, A., Inal, A.S., Suntur, B.M., Aydemir, H., Gul, S., et al. (2018). Potential Drug-Drug Interactions with Antimicrobials in Hospitalized Patients: $A$ Multicenter Point-Prevalence Study. Medical science monitor: international medical journal of experimental and clinical research, 24,4240-7. 